\title{
Convergence of Greenhouse Gas Emissions among G7 Countries
}

\author{
Ghassen El-Montasser. \\ Ecole supérieure de commerce de Tunis, University of Manouba, Tunisia \\ Roula Inglesi-Lotz* and Rangan Gupta \\ Department of Economics, University of Pretoria, South Africa
}

\begin{abstract}
The convergence of air pollutants is a major concern for policy makers since all the countries pursue the goal of allocating the emissions equally internationally in the future. Hence the examination of the existence of convergence is important for the climate change protection of the earth. In this paper, greenhouse gas (GHG) emissions convergence among the G7 countries for the period between 1990 and 2011 is examined using the pair-wise testing technique proposed by Pesaran (2007) that aims to analyse probabilistic convergence across a large number of cross section units. Next we proceed with multivariate tests for stability and the existence of unit roots. Finally, the analysis is complemented by the use of the panel stationarity test accounting for structural changes as proposed by Carrion-i-Silvestre et al. (2005) test. Overall, the results do not confirm the hypothesis of convergence for the countries in question, although, more recently, the countries have shown a small decline in their GHG emissions.
\end{abstract}

Keywords: convergence; G7 countries; GHG emissions; pair-wise testing; pollutants

\footnotetext{
${ }^{*}$ To whom correspondence should be addressed. Email: roula.inglesi-lotz@up.ac.za.
} 


\section{Introduction}

The concept of convergence dealt initially with economic growth and it was related with the reduction of inequalities between countries (Herreiras and Liu, 2013). Indeed, for more than two decades, theoretical and empirical research has focused on the study of sources of growth and convergence. There are at least three main reasons for studying real income convergence. Firstly, this exercise helps to distinguish between different growth models. Secondly, whether or not the exogenous or the endogenous version of growth is validated induces a potential for state intervention in the growth process. Thirdly, large differences were empirically observed in per capita output and in growth rates between countries over the past three decades.

Looking at environmental convergence, the path and trends of greenhouse gas emissions (GHG) and particularly of carbon dioxide $\left(\mathrm{CO}_{2}\right)$ and their geographic distribution can impact the global political economy by influencing the international negotiations and climate change agreements, as for example the United Nations Framework Convention on Climate Change (UNFCCC) 2015 in Paris. The discussions in these forums classify the countries according to their developmental status and propose policies and strategies accordingly. Especially, the Kyoto protocol has established through the years the notion that developed countries can make more effort to mitigate climate change than developing economies. This is based on the fact that developed countries have traditionally had a higher contribution to climate change and greater resources as well as the ability to pay for the environmental damages. All these assumed that the paths and capabilities of all developed countries converge and that the same or similar approaches can be adopted by all highly-industrialized countries. In the literature, as discussed in the next section, most studies focused only on the paths and possible convergence of $\mathrm{CO}_{2}$ emissions, particularly of the developed countries. 
Hence, taking into consideration the trends in the literature (convergence as an important topic), the gap of existing papers looking at the convergence of GHG emissions in total and the significance of the industrialised countries in the efforts towards climate protection policies, this paper's purpose is to investigate the convergence hypothesis of GHG emissions among the G7 countries (U.S., U.K., France, Germany, Italy, Canada and Japan).

The choice of the G7 industrialised countries is made due to their suitability for convergence analysis, as it is more possible that these countries have reached their steadystates (Romero-Avila, 2008). As Bernard and Durlauf (1996) mention "time series tests of convergence are not appropriate for those countries positioned far from the steady state as occurs with developing countries. In this case, the data would not be characterised by welldefined population moments, since the data are far from their limiting distribution”. More importantly, these are considered the most industrialised economies that as discussed in the Kyoto Protocol meetings are expected to take the lead in the fight against greenhouse gas emissions by implementing appropriate policies and using the necessary technologies

To do so, we will employ the pair-wise testing technique proposed by Pesaran (2007) that aims to analyse probabilistic convergence across a large number of cross section units. Next, following the literature (Abuaf and Jorion, 1990; Harvey and Bates, 2003), we will proceed with multivariate tests for stability and the existence of unit roots. The stationarity tests are used to conclude whether the countries have converged while the unit root tests come in handy to conclude whether the countries are converging. For the specific application, we do take into account that the greenhouse gas series may contain structural breaks due to their high connection with the current economic system and the levels of production. That is why we conclude the analysis with the panel stationarity test accounting for structural changes as proposed by Carrion-i-Silvestre, Del Barrio-Castro and López-Bazo [CBL] (2005) test. 
The paper is structured as follows. The next section presents a brief review of the literature while section 3 discusses the methodology employed and the data, followed by the empirical results. Finally, the last section concludes and presents some policy implications.

\section{Literature review}

Since the pioneering works of Baumol (1986) and Barro and Sala-i-Martin (1991, 1992), testing the convergence hypothesis involved fitting cross-country regressions. Convergence is said to occur if a negative correlation is observed between the average growth rate and initial income. Nevertheless, Quah $(1993,1996)$ criticizes this approach and recommend using time series properties to test convergence hypothesis. Likewise, Bernard and Durlauf (1996) showed that cross-country regressions cannot distinguish between assumptions regarding global or local convergence. Then Bernard and Durlauf $(1995,1996)$ propose to consider convergence as stochastic process, using the properties of time series, and test the convergence hypothesis by means of unit root tests.

The issue of convergence has recently started attracting attention in the energy and environmental literature. Studies have examined the topic of convergence among countries for various indicators such as energy intensity (Herrerias and Liu, 2013) but most often, the emissions level (for example Stazicich and List, 2003; Aldy, 2006; Westerlund and Basher, 2008; Romero-Avila, 2008; Jobert et al. 2010; Barassi et al., 2011; Herrerias, 2012; Camarero et al., 2013).

Cross-country $\mathrm{CO}_{2}$ emission convergence among 21 industrialised countries was examined in the paper by Strazicich and List (2003). Their study has proven that convergence actually exists in the years between 1960 and 1997 but their results were criticised by Li and Lin (2013) due to the study's assumption for country independence and adoption of a short sample of annual data. Aldy (2006) confirmed the same results for a sample of 23 OECD 
countries while Romero- Avila (2008) agreed by using a more advanced technique and a more recent period up to 2002.

However, the literature provide mixed evidence of the existence of convergence in emissions through the years. For example, no convergence was concluded by Aldy (2006) when the country and time sample was extended. Similarly, Lee and Chang (2008) found that only seven out of twenty-one OECD countries in their sample converge to the average emissions level. Barassi et al. (2008) also disagreed with the existence of convergence in emissions in OECD countries. Jobert et al. (2010) also confirmed the $\mathrm{CO}_{2}$ emissions existence hypothesis fort eh EU countries until 2006 although they observed slow convergence. The speed of convergence was also discussed in Barassi et al. (2011) where the convergence was proven to be achieved in particularly slow rates within 13 out of 18 OECD countries.

Although not many studies have emerged looking at the developing economies' convergence (due primarily to lack of data), Panopoulou and Pantelidis (2009) examined a sample of 128 countries for the years 1960 to 2003. They did confirm the existence of convergence in the overall time period for all countries but also for two sub-samples of countries. Li and Lin (2013) examined the topic for 110 countries over the period 1971 to 2008. Their results showed that there was convergence within subgroups of countries with similar income levels but no overall convergence was achieved.

The latest studies however, focused on the $\mathrm{CO}_{2}$ emissions only. However the importance of the Greenhouse Gas Emissions (GHG) in total is stressed at the Kyoto Protocol where the targets are not only restricted to $\mathrm{CO}_{2}$ but all the GHGs that include $\mathrm{CO}_{2}$ (carbon dioxide), $\mathrm{CH} 4$ (methane), $\mathrm{N}_{2} \mathrm{O}$ (nitrous oxide), PFCs (petrofluorocarbons), HFCs (hydrofluorocarbons) and SF6 (sulphur hexafluoride). Hence, towards global climate protection, the per capita emissions should be aimed to move towards further convergence. According to Kyoto Protocol, during the period 2008- 2012, Annex I countries committed to reduce their 
collective greenhouse gas emissions by $5.2 \%$ from the 1990 level; developing countries like China and India with larger emissions were not required to reduce emission levels (Li and Lin 2013). Developed countries are expected to take the lead in preventing global climate change, even though in less than 20 years developing countries will likely surpass them as the main emitters of $\mathrm{CO}_{2}$ (Lee and Chang 2008).

\section{Methodology}

\subsection{The pair-wise approach of Pesaran (2007)}

The Pesaran (2007)'s pair-wise testing aims to analyse probabilistic convergence across a large number of cross section units. In line with Pesaran (2007), we let $y_{i t}$ be the observed GHG series in country $\mathrm{i}$ at time $\mathrm{t}$, where $i=1, \ldots, N$ states and $t=1, \ldots, T$ time observations. The basic idea in Pesaran (2007) is to examine the stationarity properties of all $N(N-1) / 2$ gaps (also said differentials or contrasts) $g_{i j t}=y_{i t}-y_{j t}$ where $i=1, \ldots, N-1$ and $j=i+$ $1, \ldots, N$. Furthermore, consider the application of the augmented Dickey and Fuller (ADF) (1979) (or another unit root test) unit root test of order $p$ to the time series $g_{i j t}=y_{i t}-y_{j t}$, and let $Z_{i j, \tau}$ denote an indicator function that is equal to one if the corresponding unit root hypothesis is rejected at significance level $\alpha$. Pesaran (2007) studies the fraction of the $N(N$ 1)/2 gaps for which the unit-root hypothesis is rejected, and proposes a test statistic given by:

$$
\bar{Z}_{N T}=\frac{2}{N(N-1)} \sum_{i=1}^{N-1} \sum_{j=i+1}^{N} Z_{i j, \tau} .
$$

Under the null hypothesis of a unit root, the above statistic has an expected value equal to the nominal size of the underlying unit root test statistic, $\alpha$. More specifically:

$$
\lim _{T \rightarrow \infty} E\left(\bar{Z}_{N T} / H_{0}\right)=\alpha \text {. }
$$

Convergence hypothesis implies that the proportion of rejections is high and should approach $100 \%$ as $T \rightarrow \infty$ while the proportion of rejections ought to be low and around $\alpha$ in case of divergence. 


\subsection{Multivariate tests for stability and unit roots}

Multivariate tests are appropriate, if the aim is studying across a group of countries. Let $x_{t}$ be $n$ vector of contrasts between each of the $\mathrm{n}$ countries and a benchmark, e.g. $x_{t}^{\prime}=$ $\left(y_{t}^{1}, y_{t}^{2}, \ldots, y_{t}^{N}\right)$. The simplest multivariate convergence model is the zero mean $\operatorname{VAR}(1)$ process:

$$
x_{t}=\alpha x_{t-1}+\omega_{t}
$$

where $\alpha$ is a $N \times N$ matrix and $\omega_{t}$ is $N$ dimensional vector of martingale differences innovations with constant variance $\sum_{\omega}$. The model is said to be homogeneous if $\alpha=\varphi I_{N}$. Following Abuaf and Jorion (1990), Harvey and Bates (2003) propose the use of the multivariate unit root test from the homogeneous model. Specifically, they used the Waldtype statistic on $\rho=\varphi-1$, that is

$$
\psi_{0}(\mathrm{~N})=\frac{\sum_{\mathrm{t}=2}^{\mathrm{T}} \mathrm{x}_{\mathrm{t}-1}^{\prime} \widetilde{\Sigma}_{\omega}^{-1} \Delta \mathrm{x}_{\mathrm{t}}}{\left(\sum_{\mathrm{t}=2}^{\mathrm{T}} \mathrm{x}_{\mathrm{t}-1}^{\prime} \widetilde{\Sigma}_{\omega}^{-1} \mathrm{x}_{\mathrm{t}-1}\right)^{0.5}}
$$

and referred to as the multivariate homogeneous Dickey-Fuller (MHDF) statistic. $\widetilde{\Sigma}_{\omega}^{-1}$ is initially estimated by the sample covariance matrix of first-differenced data and then reestimated by iterating the estimation of $\varphi$ to convergence. Under the null hypothesis, $H_{0}: \rho=0$, $\psi_{0}(N) \stackrel{d}{\rightarrow} \frac{1}{2} \frac{\sum_{i=1}^{N}\left(W_{i}(1)^{2}-1\right)}{\left(\sum_{i=1}^{N} \int_{0}^{1} W_{i}(r)^{2} d r\right)^{0.5}}$

where $W_{i}(r)$ are independent standard Brownian motion processes, $i=1, \ldots, N$; if $N$ is large, $\quad \Psi_{0}(N)$ is approximately Gaussian. The null hypothesis is rejected when $\Psi_{0}(N)$ less than a given critical value $\delta$.

An interesting feature of the MHDF test is that it is invariant to any nonsingular transformation of $x_{t}$. Consequently, it is invariant to which country is chosen as benchmark. This feature is lost in case of heterogeneous model in which $\alpha$ is diagonal. 
One can opt for a parametric correction of the variance of the errors, through the addition of lagged differenced terms of $x_{t}$, to cope with serial correlation of the errors. The critical values of the test can be obtained from Harvey and Bates (2003); see also O'Connell (1998).

A generalization of the KPSS test can be applied to $x_{t}$ to test whether the $N$ countries have converged in the context of stability analysis. Then, the involved statistic is given by

$$
\vartheta_{0}(N)=\operatorname{Trace}\left(\widehat{\Omega}^{-1} C\right)
$$

where $C=\sum_{t=1}^{T}\left(\sum_{j=1}^{t} x_{j}\right)\left(\sum_{j=1}^{t} x_{j}\right)^{\prime}$ and $\widehat{\Omega}^{-1}$ is a non-parametric estimation of the long run variance of $x_{t}$. Under the null hypothesis of zero mean stationarity $\vartheta_{0}(n) \stackrel{d}{\rightarrow} \sum_{i=1}^{N} \int_{0}^{1} W_{i}(r)^{2} d r$, with $d$ denoting weak convergence in distribution Critical values are provided in Nyblom (1989) and Hobijn and Franses (2000).

Following Harvey and Carvalho (2002), the stationarity tests are used to conclude whether the countries have converged while the unit root tests come in handy to conclude whether the countries are converging.

\subsection{Panel stationarity test with structural changes: the Carrion-i-Silvestre, Del Barrio-} Castro and López-Bazo [CBL] (2005) test

The reasons for taking into account structural breaks in the greenhouse gas [GHG] series should be the subject of a rigorous study. It goes without saying that the GHG series is broadly correlated with the economic system. Therefore this series will be subjected to a number of structural changes. That is why, we have taken account of Carrión-i-Silvestre et al. (2005) test in the analysis of convergence. In what follows, we briefly describe the CBL (2005) test, which, by design, has the ability to test the null hypothesis of panel stationarity while allowing multiple structural breaks. It will be described as follows:

$$
x_{i, t}=c_{i}+\sum_{k=1}^{m_{i}} \theta_{i, k} D U_{i, k, t}+\beta_{i} t+\sum_{k=1}^{m_{i}} \gamma_{i, k} D T_{i, k, t}^{*}+\varepsilon_{i, t}, \ldots \ldots, t=1, \ldots \ldots, T,
$$

Where $x_{i, t}$ is the logarithm of the series GHG, $i=1 \ldots . ., N$ represents the number of cross section units and $\varepsilon_{i, t}$ is the error term. The dummy variables $D U_{i, k, t}$ and $D T_{i, k, t}^{*}$ are defined as 
$D U_{i, k, t}=1$ for $t>T_{b, k}^{i}$ and 0 otherwise, and $D T_{i, k, t}^{*}=t-T_{b, k}^{i}$ for $t>T_{b, k}^{i}$ and 0 otherwise; and $T_{b, k}^{i}$ denotes the kth date of the break for the ith individual, $k=\left\{1, \ldots m_{i}\right\}, m_{i} \geq 1$.

The model in (6) includes individual effects, individual structural break effects (i.e., shift in the mean caused by the structural breaks known as temporal effects where $\beta_{i} \neq 0$ ), and temporal structural break effects (i.e., shift in the individual time trend where $\gamma_{i} \neq 0$ ). In addition, the specification given by (6) considers several structural breaks, which are located on different unknown dates and where the number of structural breaks is allowed to vary between the panel members. The test statistic is built by running individual KPSS regression for each member of the panel and then taking the average of the $N$ individual statistics. The general expression of the test statistic is

$$
L M(\lambda)=N^{-1} \sum_{i=1}^{N}\left(\widehat{\omega}_{i}^{-2} T^{-2} \sum_{t=1}^{T} S_{i, t}^{2}\right),
$$

where $S_{i, t}=\sum_{j=1}^{t} \hat{\varepsilon}_{i, j}$ represents the partial sum process that is obtained using the estimated OLS residuals of (6), and $\widehat{\omega}_{i}^{2}$ is the consistent estimate of the long-run variance of residual $\varepsilon_{i, t}$; this allows the disturbances to be heteroscedastic across the cross-sectional dimension. In (7), $\lambda$ is defined as the vector $\lambda_{i}=\left(\lambda_{i, 1}, \ldots, \lambda_{i, m_{i}}\right)^{\prime}=\left(\frac{T_{b, 1}^{i}}{T}, \ldots, \frac{T_{b, m_{i}}^{i}}{T}\right)^{\prime}$

The test statistic for the null hypothesis of a stationary panel with multiple shifts is

$$
Z(\lambda)=\sqrt{N}(L M(\lambda)-\bar{\zeta}) / \bar{\zeta} \stackrel{d}{\rightarrow} N(0,1)
$$

As in the case of the univariate KPSS test statistic, the null hypothesis of stationarity in the panel is rejected for large values of $Z(\ddot{\mathrm{e}}) \cdot \bar{\zeta}$ and $\bar{\zeta}$ are the cross-sectional average of the individual mean and variance of $\delta_{i}(\lambda)=\widehat{\omega}_{i}^{-2} T^{-2} \sum_{t=1}^{T} S_{i, t}^{2}$.

\section{Data}

Annual data from 1990 to 2011 on GHG emissions per country were derived from the OECD Statistics databases. Data refer to total emissions of $\mathrm{CO}_{2}$ (emissions from energy use and industrial processes, e.g. cement production), $\mathrm{CH}_{4}$ (methane emissions from solid waste, 
livestock, mining of hard coal and lignite, rice paddies, agriculture and leaks from natural gas pipelines), nitrous oxide $\left(\mathrm{N}_{2} \mathrm{O}\right)$, hydrofluorocarbons (HFCs), perfluorocarbons (PFCs) and sulphur hexafluoride $\left(\mathrm{SF}_{6}\right)$. To convert them to per capita values, we employed the population series from the World Economic Outlook (WEO) database of the International Monetary Fund (IMF). In Figure 1 the raw data collected are presented. It can be observed that overall the seven countries do not exhibit convergence characteristics for the specific time period.

Figure 1: GHG emissions per capita of G7 countries (1990-2011)

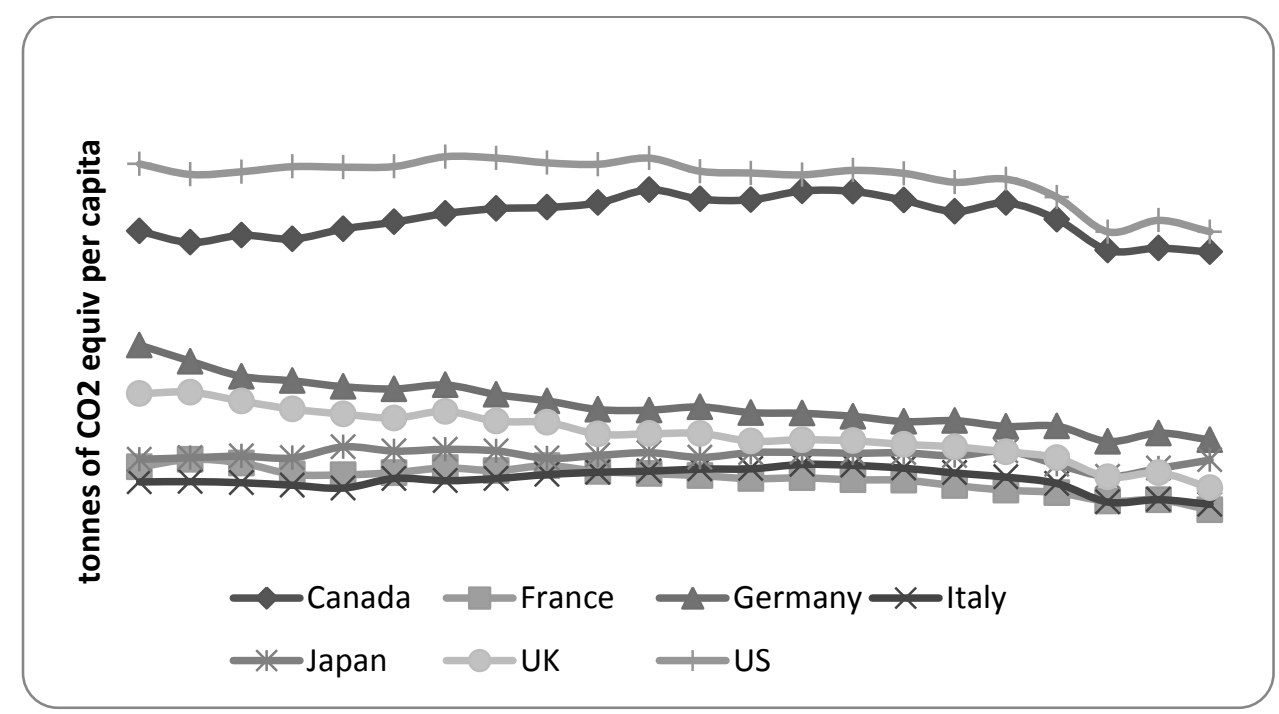

Sources: OECD Statistics and IMF

\section{Empirical results}

Before presenting the results of the ADF and KPSS tests, we need to provide some details regarding their corresponding specifications. For the KPSS test, we did not take into account the constant while testing for zero-mean stationarity of differences between the two series in question. It has been shown that the KPSS test without constant has power against a stationary process with a non-zero mean, as well as, against a non-stationary process (Busetti and Harvey, 2002). Hence, we applied the KPSS test without the constant. In this way, we 
test if the series converged individually or in groups, and if the involved convergence is absolute. When we test for conditional (relative) convergence by recourse to the ADF, we have replaced the mean of the differences between the two series by the last value of the differenced series to test whether our data are converging. According to Busetti et al. (2006), the subtraction of the last observation is a possible way of enhancing the power of the ADF test. Also, we have chosen 5 as a maximum number of delayed terms.

The results of the ADF and KPSS tests applied to different pair-wise GHG differentials are presented respectively in Tables 1 and 2.

Table 1: Results of pair-wise ADF statistics for GHG series

\begin{tabular}{|c|c|c|c|c|c|c|c|c|}
\hline & \multicolumn{4}{|c|}{ ADF-NO INTERCEPT } & \multicolumn{4}{|c|}{ ADF-WITH INTERCEPT } \\
\hline & Statistics & $\varphi$ & Reject & Lags & Statistics & $\varphi$ & Reject & Lags \\
\hline CAN-FR & 1.7215 & 1.0529 & - & 1 & -2.0579 & 0.8831 & - & 1 \\
\hline $\begin{array}{l}\text { CAN- } \\
\text { GER }\end{array}$ & -5.1356 & 0.92 & $1 \%$ & 1 & -3.8652 & 0.8586 & $1 \%$ & 1 \\
\hline CAN-IT & 3.0227 & 1.0640 & - & 1 & -0.9060 & 0.9273 & - & 1 \\
\hline $\begin{array}{l}\text { CAN- } \\
\text { JAP }\end{array}$ & -1.5563 & 0.9872 & - & 1 & -1.8987 & 0.8776 & - & 1 \\
\hline CAN-UK & -0.4368 & 0.9745 & - & 2 & -2.6190 & 0.9028 & - & 1 \\
\hline CAN-US & -2.1359 & 0.9973 & $5 \%$ & 1 & -1.0902 & 0.9115 & - & 1 \\
\hline FR-GER & -0.9124 & 0.9920 & - & 1 & -1.6363 & 0.8416 & - & 1 \\
\hline FR-IT & -2.1110 & 0.8073 & $5 \%$ & 1 & -1.7272 & 0.7975 & - & 1 \\
\hline FR-JAP & 1.0471 & 1.0106 & - & 1 & -2.4134 & 0.3478 & - & 1 \\
\hline FR-UK & -2.3083 & 0.9509 & $5 \%$ & 1 & -1.0372 & 0.9136 & - & 1 \\
\hline FR-US & 2.8255 & 1.0065 & - & 2 & -2.4163 & 0.7881 & - & 2 \\
\hline GER-IT & -1.5995 & 0.9843 & - & 1 & -1.9150 & 0.8783 & - & 1 \\
\hline GER-JA & 1.5509 & 1.0551 & - & 1 & -2.4308 & 0.7675 & - & 1 \\
\hline GER-UK & 0.8701 & 1.0073 & - & 1 & -0.5843 & 0.8202 & - & 1 \\
\hline GER-US & 2.2695 & 1.0084 & - & 1 & -3.7687 & 0.8418 & $5 \%$ & 1 \\
\hline IT-JAP & 0.5687 & 1.0049 & - & 1 & -0.9572 & 0.7686 & - & 1 \\
\hline IT-UK & -3.5581 & 0.9191 & $1 \%$ & 1 & -1.9181 & 0.8831 & - & 1 \\
\hline IT-US & 1.7415 & 1.0035 & - & 1 & -1.1563 & 0.8095 & - & 1 \\
\hline JAP-UK & 2.5101 & 1.0335 & - & 1 & -1.0082 & 0.8785 & - & 1 \\
\hline JAP-US & 0.7411 & 1.0025 & - & 1 & -1.7606 & 0.7845 & - & 1 \\
\hline UK-US & 4.0708 & 1.0112 & - & 1 & -2.6660 & 0.8914 & $10 \%$ & 1 \\
\hline
\end{tabular}


Table 2: KPSS test without constant

\begin{tabular}{|l|l|l|l|}
\hline & Statistics & pvalue & Reject \\
\hline CAN-FR & 2.0572 & 0.01 & $5 \%$ \\
\hline CAN-GER & 2.0703 & 0.01 & $5 \%$ \\
\hline CAN-IT & 1.8814 & 0.01 & $5 \%$ \\
\hline CAN-JAP & 1.9986 & 0.01 & $5 \%$ \\
\hline CAN-UK & 2.0918 & 0.01 & $5 \%$ \\
\hline CAN-US & 2.0070 & 0.01 & $5 \%$ \\
\hline FR-GER & 1.7580 & 0.01 & $5 \%$ \\
\hline FR-IT & 1.5269 & 0.01 & $10 \%$ \\
\hline FR-JAP & 0.8209 & 0.01 & - \\
\hline FR-UK & 1.9481 & 0.01 & $5 \%$ \\
\hline FR-US & 1.9249 & 0.01 & $5 \%$ \\
\hline GER-IT & 1.8803 & 0.01 & $5 \%$ \\
\hline GER-JAP & 1.8634 & 0.01 & $5 \%$ \\
\hline GER-UK & 0.2061 & 0.10 & - \\
\hline GER-US & 2.0293 & 0.01 & $5 \%$ \\
\hline IT-JAP & 0.4232 & 0.0672 & - \\
\hline IT-UK & 2.0071 & 0.01 & $5 \%$ \\
\hline IT-US & 1.1160 & 0.01 & $5 \%$ \\
\hline JAP-UK & 1.8326 & 0.01 & $5 \%$ \\
\hline JAP-US & 1.7271 & 0.01 & $5 \%$ \\
\hline UK-US & 2.0650 & 0.01 & $5 \%$ \\
\hline
\end{tabular}

The rejection frequencies of unit root hypothesis for $5 \%$ and $10 \%$ nominal levels are shown in Table 3. Clearly, we can conclude that GHG series in the G7 group diverged using Pesaran's (2007) pair-wise approach since the rejection frequencies are somewhat low.

Table 3: The fraction of rejection $\bar{Z}_{N T}$ of Pesaran (2007)

\begin{tabular}{|l|l|l|}
\hline & $5 \%$ & $10 \%$ \\
\hline ADF- no intercept & $23.81 \%$ & $23.81 \%$ \\
\hline ADF with intercept & $9.52 \%$ & $14.29 \%$ \\
\hline KPSS & $19.05 \%$ & $14.29 \%$ \\
\hline
\end{tabular}

With regard to multivariate tests, we subtracted from each individual series the crosssection mean. This can be beneficial especially in two ways: First, the cross-section mean series can be regarded as a benchmark, and then, the study of all individual series is guaranteed. Second, this subtraction can mitigate the effects of not taking into account the cross-sectional dependence, reflecting reality, since the analysis of macroeconomic time series for different countries are affected by similar events that could introduce dependency between individuals in the panel data set. We follow Levin et al, (Levin, Lin and Chu [LLC]) (2002), who suggested removing the cross-section mean which is equivalent to include 
temporal effects in the panel data. We applied the LLC test to GHG series to compare its results with those from the other multivariate homogeneous test, videlicet, the MHDF test.

Given that the MHDF test does not take account of heterogeneity, we decided to add the Im et al. (Im, Pesaran and Shin [IPS]) (2003) test that has been formulated by allowing for this assumption. Similarly, we included the panel stationarity test of Hadri (2000) allowing for heterogeneity. As far as convergence is concerned, this may be beneficial since the homogeneity assumption restricts every country to converge at the same rate.

Table 4: $p$-values of panel and multivariate unit root tests

\begin{tabular}{|l|l|l|}
\hline $\begin{array}{l}\text { Multivariate unit root } \\
\text { tests }\end{array}$ & $\begin{array}{l}\text { Conditional } \\
\text { convergence }\end{array}$ & $\begin{array}{l}\text { Absolute } \\
\text { convergence }\end{array}$ \\
\hline MHDF & 0.9383 & 0.9892 \\
\hline LLC $\left(\right.$ Adjusted $\left.t^{*}\right)$ & 0.0000 & 0.9763 \\
\hline IPS(Ztbar) & 0.2258 & - \\
\hline
\end{tabular}

The results of panel and multivariate homogeneous unit root tests are presented in Table 4. As we can see from this table, most tests do not reject the null hypothesis of unit roots in the whole panel, and therefore the convergence hypothesis is not verified. The only exception to this observation is the modified student statistic of LLC (2002), when only the level is taken into account in the deterministic component. The null hypothesis is rejected in this case. This result contradicts the conclusion that we can draw from the same statistic from the model with no deterministic term. This contradiction could be due to individual effects. Meanwhile from Table 5, we can deduce that all tests of panel stationarity reject the null hypothesis of stationarity. This also includes the CBL test, which rejects the null hypothesis of stationarity with structural breaks (for evidence on individual country structural breaks, see Table A1 in the Appendix).

Table 5: Results of panel and multivariate stationarity tests

\begin{tabular}{|l|l|l|}
\hline & \multicolumn{2}{|c|}{ Absolute convergence } \\
\hline Multivariate stationarity tests & Statistics & Pvalues \\
\hline MKPSS & 832 & 0.0000 \\
\hline Hadri (2000)'s test & 30.0926 & 0.0000 \\
\hline CBL Test & 1638.6108 & 0.0000 \\
\hline
\end{tabular}




\section{Conclusion}

The convergence of air pollutants is a major worry for policy makers since all the countries pursue the goal of allocating the emissions equally internationally in the future. Hence the examination of the existence of convergence is important for the climate change protection of the earth. This paper's purpose is to investigate the convergence hypothesis of GHG emissions among the G7 countries (U.S., U.K., France, Germany, Italy, Canada and Japan). This group of industrialised countries is among those that have committed for high reductions of their emissions by 2020 (compared to 1990 levels) according to the Kyoto Protocol.

Firstly, we used the pair-wise testing technique proposed by Pesaran (2007) followed by multivariate tests for stability and the existence of unit roots. For the specific application of GHG emissions, we do take into account that the GHG series may contain structural breaks. Hence, we finish off the analysis with the panel stationarity test accounting for structural changes as proposed by Carrion-i-Silvestre et al., (2005), as well as standard panel unit root tests of Levin et al. (2002) and Im et al. (2003).

Overall, the results do not confirm the hypothesis of convergence for the countries in question from 1990 to 2011, although the countries have shown a certain small decline in their GHG emissions. This can be interpreted by two factors: first, the structural changes could be rather country-specific, and thus, we do not expect that it favor a possible convergence of GHG series in the G7. Second, even though these structural changes have common causes to all countries, they may not have a significant effect to promote convergence.

In general, similar countries with respect to preferences and technology tend to converge (McKibbin and Stegman, 2005). Here, although the G7 countries are considered the strongest economies internationally and among the leaders in adoption of technologies towards 
improving the environment, they still have significant differences. Evidence for this fact can also be seen from the differences in the year of structural changes in each economy. Hence, the assumption of absolute convergence is not reflective of the reality.

McKibbin and Stegman (2005) have noted that one of the reasons for the considering the existence of emissions convergence a crucial topic for researchers and policy makers is the ability for projections. Future environmental policies will be better founded if convergence (even conditional) exists. According to our results, the projection for the G7 economies' GHG emissions cannot be estimated with precision. As a result the policies, although in a concerted effort, should also be country-specific and will depend on the socioeconomic characteristics of each country.

In recent years and through the Kyoto agreements, a significant difference between developed and developing economies was observed both with regards to the actual emissions but also their policies towards their reduction. A possible convergence among the developed countries, such as the G7, could have altered the behavior of developing countries and lead to a common strategy in the future. The non-convergence in the big industrialized countries shows that the global energy system is not mature enough to incorporate internationallyimplemented, common policies and strategies; fact that affects the developing nations that lag behind. Convergence, hence, or lack thereof, should be taken into account in the discussions at the United Nations Framework Convention on Climate Change (UNFCCC) 2015 in Paris where the international leaders will discuss on a common environmental future.

In conclusion, the overall economic policy suggestion that can be derived from the results of this paper is that only regional, country-specific policies and strategies (and not umbrella programmes for all) that will incorporate environmental harmonization policies will assist in reducing the emissions levels, at this stage. Strict overall international regulations that are 
usually an aftereffect of high levels of policy integration are not the current solution for the

G7 economies.

\section{References}

Abuaf, N. and Jorion, P. 1990. Purchasing power parity in the long run. Journal of Finance 45, 157-74.

Aldy, J.E., 2006. Per capita carbon dioxide emissions: convergence or divergence? Environmental Resource Economics 33, 533-555.

Barassi, M.R., Cole, M.A., Elliott, R.J.R., 2008. Stochastic divergence or convergence of per capita carbon dioxide emissions: re-examining the evidence. Environmental and Resource Economics 40, 121-137.

Barassi, M.R., Cole, M.A., Elliott, R.J.R., 2011. The stochastic convergence of CO2 emissions: a long memory approach. Environmental and Resource Economics 49, 367-385.

Barro, R., and Sala-i-Martin, X., 1991. Convergence across States and Regions, Brooking Papers on Economic Activity, 1: 107-182

Barro, R., and Sala-i-Martin, X., 1995. Economic Growth, Mc Graw Hill, New-York

Baumol, W., 1986. Productivity Growth, Convergence and Welfare : What the Long Run Data Show, American Economic Review, 76, 1072-1085

Bernard A., Durlauf S. 1996. Interpreting tests of the convergence hypothesis. Journal of Econometrics, 71, 161-173.

Bernard A., Durlauf S. 1995. Convergence in international output. Journal of Applied Econometrics, 10, 97-108.

Bernard, A.D., Durlauf, S.N., 1996. Interpreting tests of the convergence hypothesis. Journal of Econometrics 71, 161-173.

Busetti, F. and A.C. Harvey, 2008. Testing For Trend, Econometric Theory, 24, 72-87.

Busetti, F., S.Fabiani and A.C. Harvey, 2006. Convergence of prices and rates of inflation, Oxford Bulletin of Economics and statistics, 68, 863-877.

Camareroa, M., Picazo-Tadeo, B., Tamarit, C. 2013. Are the determinants of CO2 emissions converging among OECD countries? Economics Letters 118, 159-162.

Carrion-i-Silvestre, J.L., Del Barrio-Castro, T. and Lopez-Bazo, E., 2005, Breaking the panels: an application to the GDP per capita, Econometrics Journal 8, 159-175.

Ezcurra R. 2007. Is there cross-country convergence in carbon dioxide emissions? Energy Policy 35,1363-72.

Hadri, K. 2000. Testing for Unit Roots in Heterogeneous Panel Data, Econometrics Journal, 3, 148-161.

Harvey, A.C. and D. Bates 2003. Multivariate unit root tests and testing for convergence, University of Cambridge, mimeo.

Harvey, A.C. and V. Carvalho 2002. Models for converging economies, University of Cambridge - DAE Working Papers.

Herrerias, M.J. 2012. CO2 weighted convergence across the EU-25 countries (19202007). Applied Energy 92, 9-16

Herrerias, M.J., Liu, G. 2013.Electricity intensity across Chinese provinces: New evidence on convergence and threshold effects. Energy Economics 36, 268-276

Hobjin B. and P.H. Franses, 2000. Asymptotically perfect and relative convergence of productivity, Journal of Applied Econometrics 15, 59-81.

Im, K. S., Pesaran, M. H. and Shin, Y. 2003. Testing for unit roots in heterogeneous panels. Journal of Econometrics 115, 53-74. 
Jobert, Th., Karanfil, F., Tykhonenko, A., 2010. Convergence of per capita carbon dioxide emissions in the EU: legend or reality? Energy Economics 32,1364-1373.

Lee, C.-C., Chang, C.-P., 2008. New evidence on the convergence of per capita carbon dioxide emissions from panel seemingly unrelated regressions augmented Dickey- Fuller tests. Energy 33, 1468-1475.

Li, X., Lin, B. 2013. Global convergence in per capita CO2 emissions. Renewable and Sustainable Energy Reviews 24, 357-363.

McKibbin, W.J., Stegman, A. 2005. Convergence and per capita carbon emissions. Brooking Discussion papers in International Economics No 167. Available at http://www.brookings.edu/ /media/research/files/papers/2005/5/globalenvironment\%20steg man/200505_bdpie167.pdf

Meng, M., Payne, J.E., Lee, J. 2013. Convergence in per capita energy use among OECD countries. Energy Economics 36, 536-545.

Müller, U. K. and Elliott, G. 2003. Tests for unit roots and the initial condition. Econometrica

Nyblom, J. 1989. Testing for the Constancy of Parameters over Time, Journal of the American Statistical Association, 84, 223-30.

O'Connell, P., 1998. The overvaluation of purchasing power parity. Journal of International Economics 44, 1-19.

Panopoulou E., Pantelidis, T., 2009. Club convergence in carbon dioxide emissions. Environmental and Resource Economics 44, 47-70.

Pesaran, M.H., 2007. A pair-wise approach to testing for output and growth convergence. Journal of Econometrics 138, 312-355.

Quah D. 1993. Galton's fallacy and tests of the convergence hypothesis", Scandinavian Journal of Economics, 95, 427-443.

Quah, D., 1996. Empirics for economic growth and convergence. European Economic Review 40, 1353-1375.

Romero-Ávila, D., 2008. Convergence in carbon dioxide emissions among industrialized countries revisited. Energy Economics 30, 2265-2282.

Strazicich, M.C., List, J.A., 2003. Are CO2 emission levels converging among industrial countries? Environmental and Resource Economics 24, 263-271.

Westerlund, J., Basher, S.A., 2008. Testing for convergence in carbon dioxide emissions using a century of panel data. Environmental and Resource Economics 40 (1), 109-120.

Table A1: Detection of breaks

\section{Appendix}

\begin{tabular}{|l|l|l|l|l|}
\hline Country & Date 1 & Date 2 & Date 3 & Date 4 \\
\hline Canada & 1993 & 1996 & 1999 & 2008 \\
\hline France & 1992 & 1995 & 2005 & 2008 \\
\hline Germany & 1992 & 1997 & 2004 & 2008 \\
\hline Italy & 1997 & 2002 & 2005 & 2008 \\
\hline Japan & 1993 & 1997 & 2001 & 2007 \\
\hline United Kingdom & 1992 & 1998 & 2005 & 2008 \\
\hline United States & 1992 & 1995 & 1999 & 2008 \\
\hline G7 & 1992 & 1995 & 1999 & 2004 \\
\hline
\end{tabular}

